\title{
Interpreting operando XANES of Surface-supported Subnanometer Clusters: When Fluxionality, Oxidation State and Size Effect Fight
}

Borna Zandkarimi, ${ }^{a}$ Geng Sun, ${ }^{b}$ Avik Halder, ${ }^{c}$ Soenke Seifert, ${ }^{d}$ Stefan Vajda,, e, f Philippe Sautet, ${ }^{a, b, g^{*}}$ and Anastassia N. Alexandrova ${ }^{a, g^{*}}$

aDepartment of Chemistry and Biochemistry, University of California, Los Angeles, Los Angeles, CA 90095

${ }^{\mathrm{b}}$ Department of Chemical and Biomolecular Engineering, University of California, Los Angeles, Los Angeles, California 90095, United States

${ }^{c}$ Materials Science Division, Argonne National Laboratory, Argonne Illinois 60439, United States

${ }^{\mathrm{d} X-r a y ~ S c i e n c e ~ D i v i s i o n, ~ A r g o n n e ~ N a t i o n a l ~ L a b o r a t o r y, ~} 9700$ South Cass Avenue, Argonne, Illinois 60439, United States

${ }^{\mathrm{e}}$ Institute for Molecular Engineering, The University of Chicago, 5640 South Ellis Avenue, Chicago, Illinois 60637, USA

fDepartment of Nanocatalysis, J. Heyrovský Institute of Physical Chemistry, Czech Academy of Sciences, Dolejškova 3, 18223Prague 8, Czech Republic gCaliforniaNanoSystems Institute, 570 Westwood Plaza, Los Angeles, CA 90095

*Corresponding Authors: Philippe Sautet, (310) 825-8485, sautet@ucla.edu, Anastassia N. Alexandrova, (310) 825-3769, ana@,chem.ucla.edu 


\section{SUPPORTING INFORMATION}

\section{Table of content:}

Figure S1. Obtained UNCD planar-averaged potential curve as a function of $\mathrm{z}$.

Figure S2. The effect of Hubbard $U$ on the XANES spectra of $\mathrm{Cu}_{5} \mathrm{O}_{5} / \mathrm{UNCD}$ and $\mathrm{Cu}_{5} \mathrm{O}_{3} / \mathrm{UNCD}$.

Figure S3. Experimental XANES of $\mathrm{Cu}_{4} \mathrm{O}_{\mathrm{x}} / \mathrm{Al}_{2} \mathrm{O}_{3}$ at $423 \mathrm{~K}, 673 \mathrm{~K}$, and $773 \mathrm{~K}$ along with their corresponding LCF spectrum.

Figure S4. Horizontal GISAXS cuts from supported $\mathrm{Cu}_{4}$ clusters on alumina under a flow of $2 \%$ propane and $2 \%$ oxygen in helium under a pressure of $1.1 \mathrm{~atm}$.

Table S1. Rising-edge and white line peak of bulk $\mathrm{Cu}, \mathrm{Cu}_{2} \mathrm{O}, \mathrm{CuO}, \mathrm{Cu}_{5} \mathrm{O}_{5} / \mathrm{UNCD}, \mathrm{Cu}_{5} \mathrm{O}_{3} / \mathrm{UNCD}$, and $\mathrm{Cu}_{4} \mathrm{O}_{\mathrm{x}} / \mathrm{Al}_{2} \mathrm{O}_{3}(\mathrm{x}=2-5)$ obtained from finite difference method (FDM) implemented in FDMNES software.

Table S2. Obtained coefficients from the LCF of calculated XANES for $\mathrm{Cu}_{5} \mathrm{O}_{5} / \mathrm{UNCD}$, $\mathrm{Cu}_{5} \mathrm{O}_{3} / \mathrm{UNCD}$, and $\mathrm{Cu}_{4} \mathrm{O}_{\mathrm{x}} / \mathrm{Al}_{2} \mathrm{O}_{3}(\mathrm{x}=2-5)$ to the bulk $\mathrm{Cu}_{2} \mathrm{O}$ and $\mathrm{CuO}$ XANES, for three different isomers.

Table S3. Obtained coefficients of $\mathrm{Cu}_{4} \mathrm{O}_{\mathrm{x}} / \mathrm{Al}_{2} \mathrm{O}_{3}(\mathrm{x}=2-5)$ from LCF to the experimental XANES at $423 \mathrm{~K}, 673 \mathrm{~K}$, and $773 \mathrm{~K}$.

Table S4. Energies of $\mathrm{Cu}_{4} \mathrm{O}_{\mathrm{x}} / \mathrm{Al}_{2} \mathrm{O}_{3}(\mathrm{x}=2-5), \mathrm{Cu}_{5} \mathrm{O}_{5} / \mathrm{UNCD}$, and $\mathrm{Cu}_{5} \mathrm{O}_{3} / \mathrm{UNCD}$ obtained from PES sampling. 


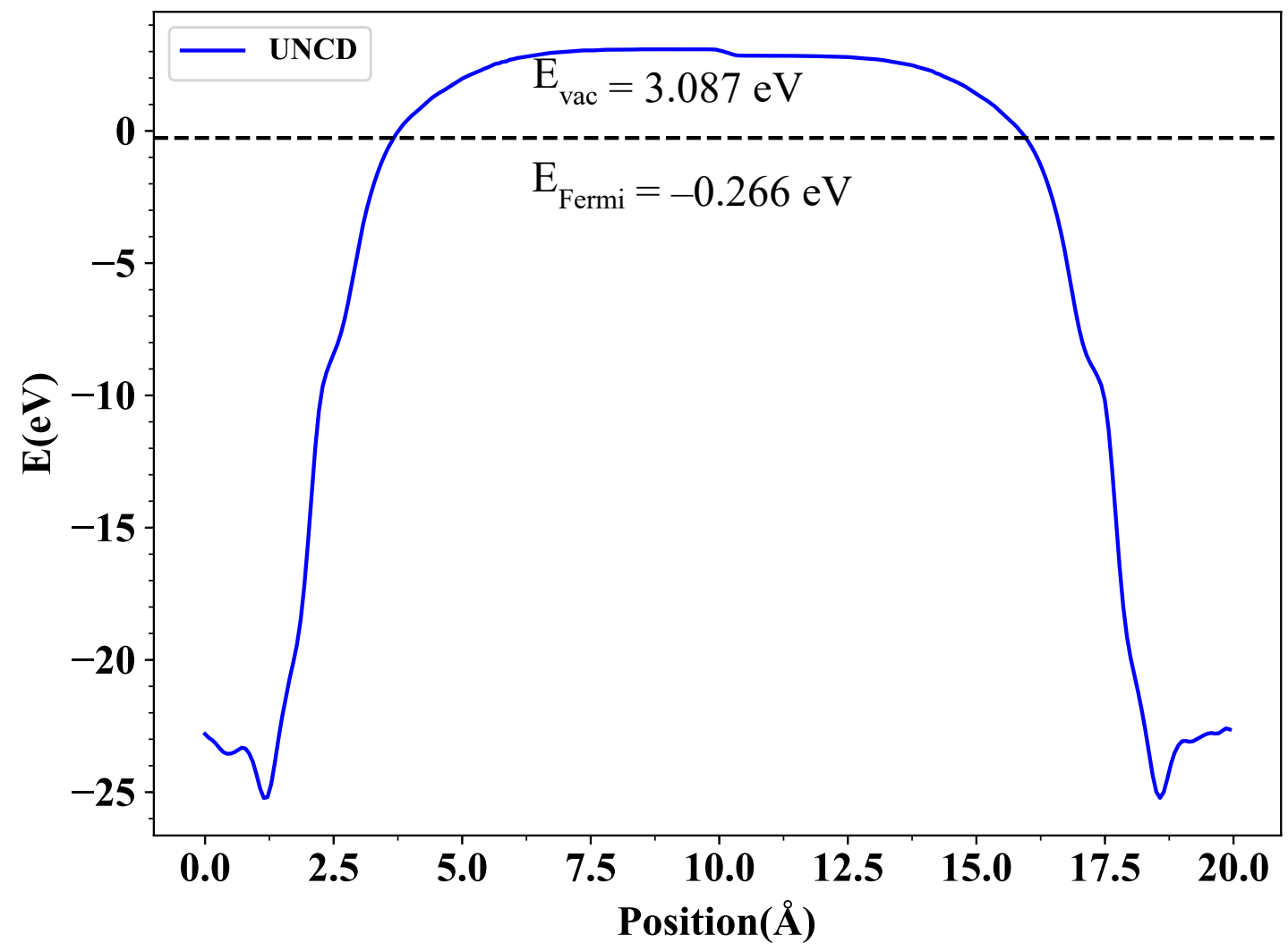

Figure S1. Obtained UNCD planar-averaged potential curve as a function of $z$. Work function is obtained as the difference between the vacuum potential and Fermi level. 


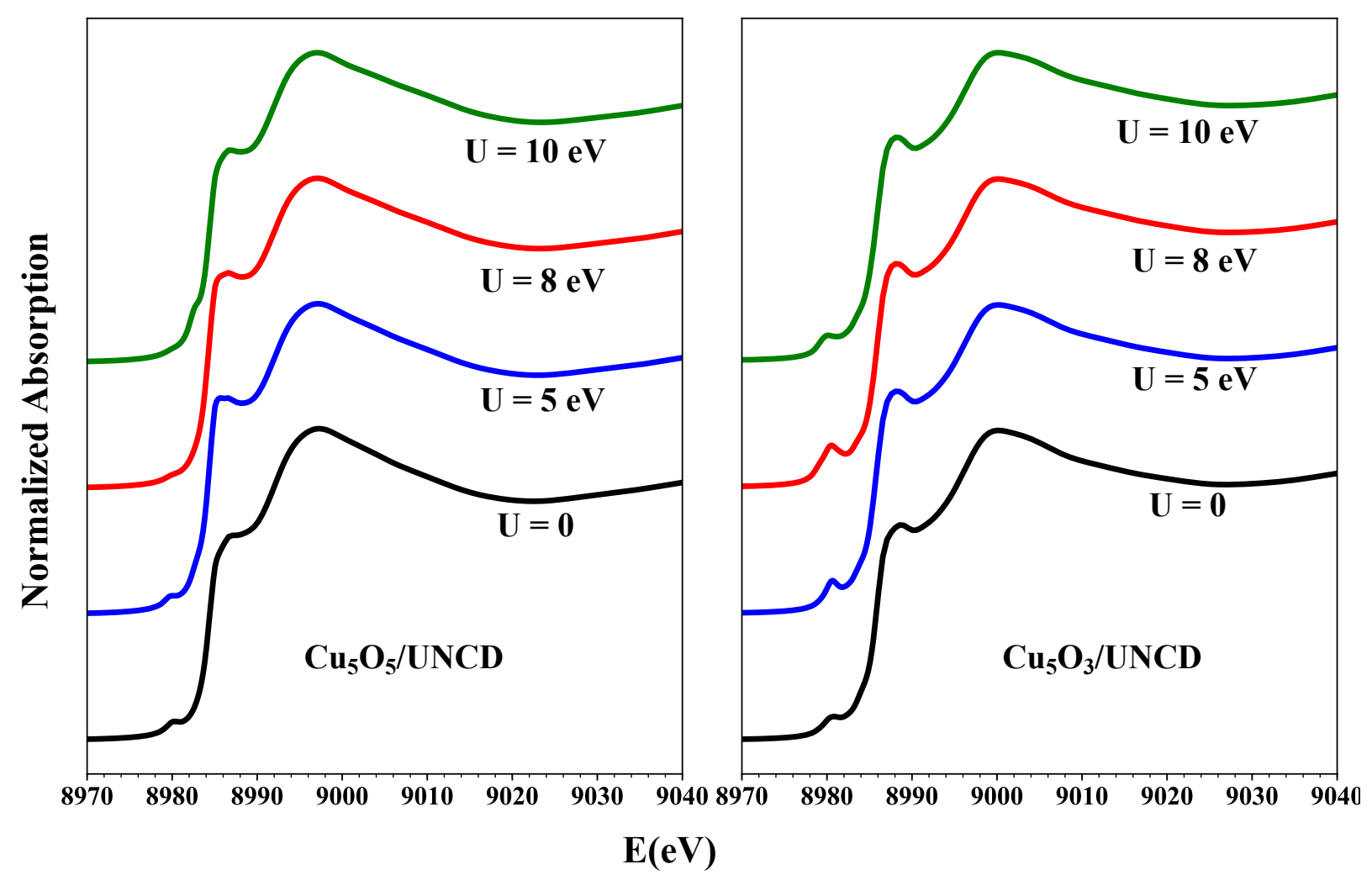

Figure S2. The effect of Hubbard $U$ on the XANES spectra of $\mathrm{Cu}_{5} \mathrm{O}_{5} / \mathrm{UNCD}$ and $\mathrm{Cu}_{5} \mathrm{O}_{3} / \mathrm{UNCD}$. 


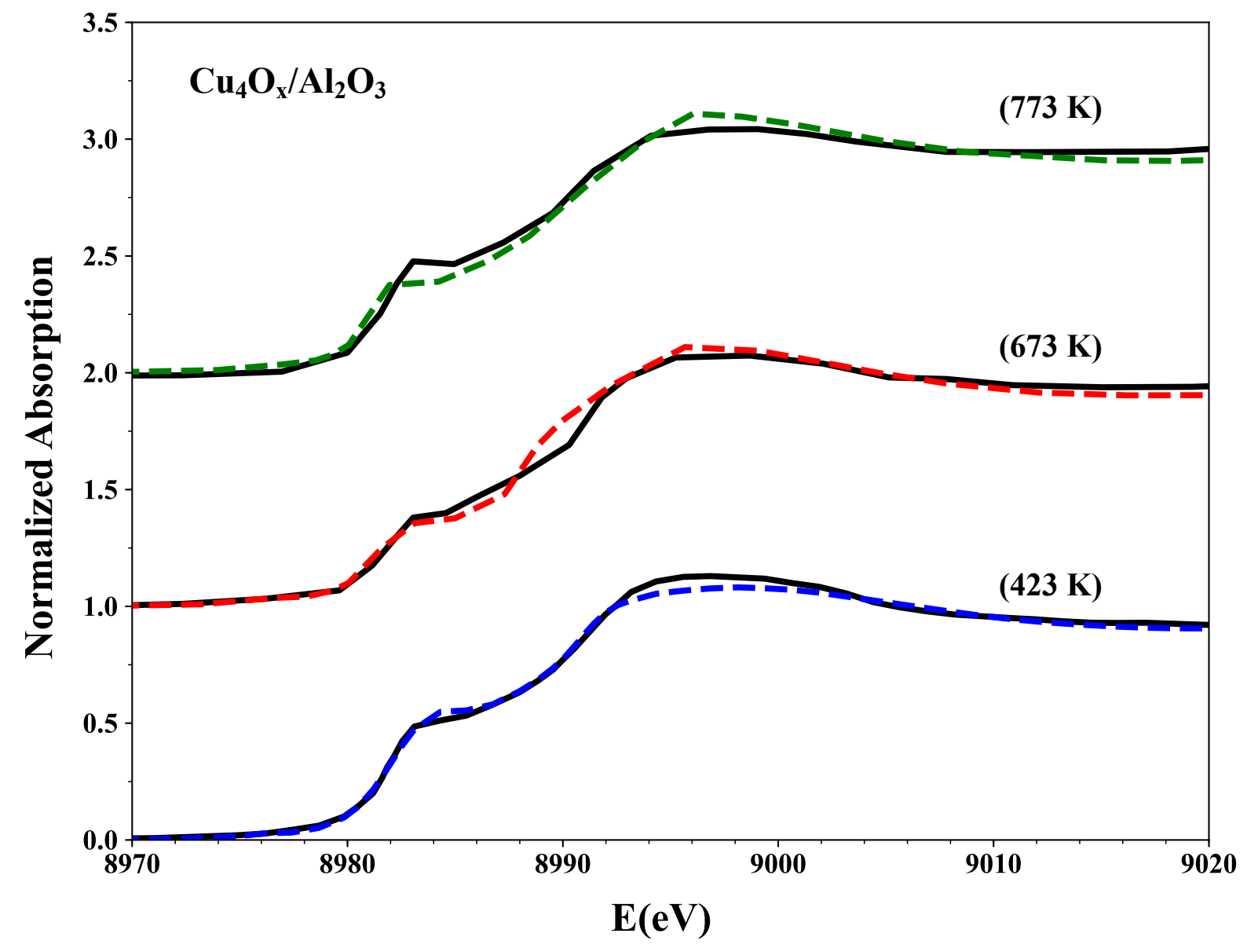

Figure S3. Experimental XANES of $\mathrm{Cu}_{4} \mathrm{O}_{\mathrm{x}} / \mathrm{Al}_{2} \mathrm{O}_{3}$ at $423 \mathrm{~K}, 673 \mathrm{~K}$, and $773 \mathrm{~K}$ (solid curves) along with LCF (dashed curves) obtained by fitting to $\mathrm{Cu}_{4} \mathrm{O}_{\mathrm{x}} / \mathrm{Al}_{2} \mathrm{O}_{3}(\mathrm{x}=2-5)$ structures computed from grand canonical global optimization. 


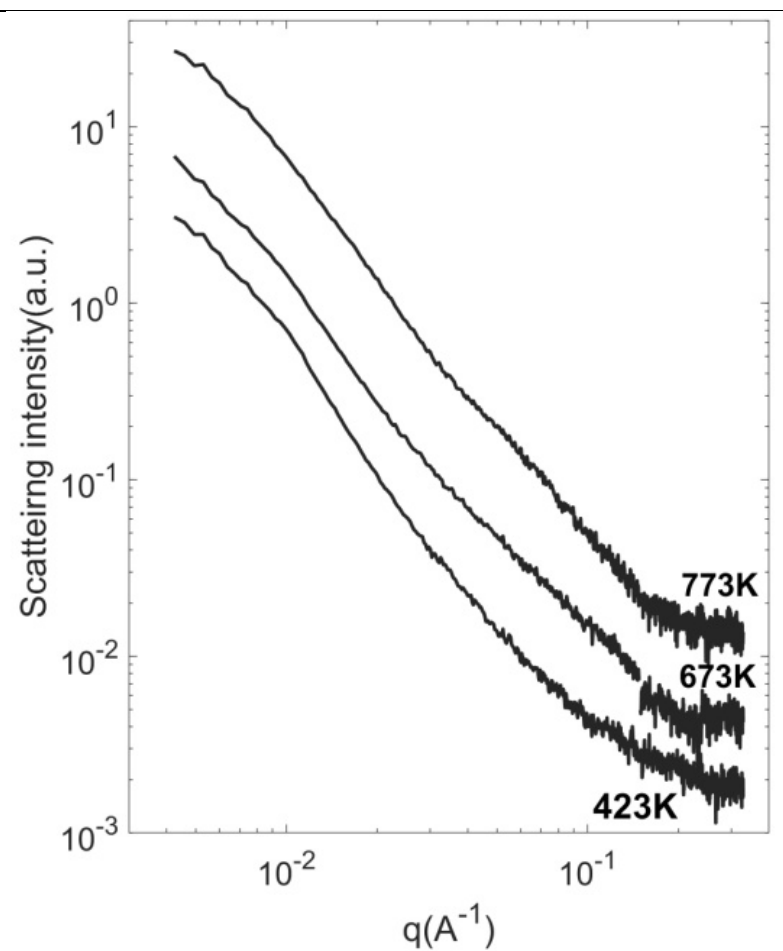

Figure S4. Horizontal GISAXS cuts from supported $\mathrm{Cu}_{4}$ clusters on alumina under a flow of $2 \%$ propane and $2 \%$ oxygen in helium under a pressure of $1.1 \mathrm{~atm}$. The spectra were collected at $9.1 \mathrm{keV}$. No evidence of agglomeration seen at any of the measured temperatures.

Table S1. Energies corresponding to the rising-edge and white line peak of bulk $\mathrm{Cu}, \mathrm{Cu}_{2} \mathrm{O}, \mathrm{CuO}$, $\mathrm{Cu}_{5} \mathrm{O}_{5} / \mathrm{UNCD}, \mathrm{Cu}_{5} \mathrm{O}_{3} / \mathrm{UNCD}$, and $\mathrm{Cu}_{4} \mathrm{O}_{\mathrm{x}} / \mathrm{Al}_{2} \mathrm{O}_{3}(\mathrm{x}=2-5)$ obtained from finite difference method (FDM). Note that for $\mathrm{Cu}_{4} \mathrm{O}_{\mathrm{x}} / \mathrm{Al}_{2} \mathrm{O}_{3}(\mathrm{x}=2-5)$ the three lowest energy isomers with significantly different geometries (A, B, and C) were chosen.

\begin{tabular}{|c|c|c|c|c|c|c|}
\hline Structure & \multicolumn{3}{|c|}{$E_{\text {rising-edge }}(\mathrm{eV})$} & \multicolumn{3}{|c|}{$\mathbf{E}_{\text {white-line }}(\mathrm{eV})$} \\
\hline $\mathrm{Cu}$ & \multicolumn{3}{|c|}{8983.0} & \multicolumn{3}{|c|}{8994.0} \\
\hline $\mathrm{Cu}_{2} \mathrm{O}$ & \multicolumn{3}{|c|}{8984.0} & \multicolumn{3}{|c|}{8996.5} \\
\hline $\mathrm{CuO}$ & \multicolumn{3}{|c|}{8986.0} & \multicolumn{3}{|c|}{8997.0} \\
\hline Isomer & $\mathbf{A}$ & B & $\mathrm{C}$ & A & B & $\mathrm{C}$ \\
\hline $\mathrm{Cu}_{5} \mathrm{O}_{3} / \mathrm{UNCD}$ & 8988.6 & 8984.1 & 8988.4 & 9000.0 & 8998.6 & 9001.4 \\
\hline $\mathrm{Cu}_{5} \mathrm{O}_{5} / \mathrm{UNCD}$ & 8986.6 & 8987.9 & 8987.3 & 8997.0 & 8999.4 & 8998.5 \\
\hline $\mathrm{Cu}_{4} \mathrm{O}_{2} / \mathrm{Al}_{2} \mathrm{O}_{3}$ & 8987.2 & 8990.3 & 8989.3 & 8999.7 & 8997.3 & 8999.3 \\
\hline $\mathrm{Cu}_{4} \mathrm{O}_{3} / \mathrm{Al}_{2} \mathrm{O}_{3}$ & 8988.2 & 8989.1 & 8987.6 & 8999.7 & 8999.1 & 9000.6 \\
\hline
\end{tabular}




$\begin{array}{lllllll}\mathrm{Cu}_{4} \mathrm{O}_{4} / \mathrm{Al}_{2} \mathrm{O}_{3} & 8988.1 & 8987.9 & 8988.9 & 8998.1 & 8999.4 & 9001.4 \\ \mathrm{Cu}_{4} \mathrm{O}_{5} / \mathrm{Al}_{2} \mathrm{O}_{3} & 8988.8 & 8988.0 & 8988.7 & 8998.3 & 9000.0 & 9002.2\end{array}$

Table S2. LCF of calculated XANES for $\mathrm{Cu}_{5} \mathrm{O}_{5} / \mathrm{UNCD}, \mathrm{Cu}_{5} \mathrm{O}_{3} / \mathrm{UNCD}$, and $\mathrm{Cu}_{4} \mathrm{O}_{\mathrm{x}} / \mathrm{Al}_{2} \mathrm{O}_{3}(\mathrm{x}=2-5)$ to the bulk $\mathrm{Cu}_{2} \mathrm{O}$ and $\mathrm{CuO}$ XANES, for three different isomers. $\mathrm{C}_{\mathrm{Cu} 2 \mathrm{O}}$ and $\mathrm{C}_{\mathrm{CuO}}$ are the coefficients of $\mathrm{Cu}_{2} \mathrm{O}$ and $\mathrm{CuO}$, respectively, in the LCF of each surface-supported cluster.

\begin{tabular}{ccccccc}
\hline \multirow{2}{*}{ Structure/Isomer } & \multicolumn{2}{c}{$\mathbf{A}$} & \multicolumn{3}{c}{ B } \\
\cline { 2 - 7 } & $\mathrm{C}_{\mathrm{Cu} 2 \mathrm{O}}$ & $\mathrm{C}_{\mathrm{CuO}}$ & $\mathrm{C}_{\mathrm{Cu} 2 \mathrm{O}}$ & $\mathrm{C}_{\mathrm{CuO}}$ & $\mathrm{C}_{\mathrm{Cu} 2 \mathrm{O}}$ & $\mathrm{C}_{\mathrm{CuO}}$ \\
\hline $\mathrm{Cu}_{5} \mathrm{O}_{5} / \mathrm{UNCD}$ & 0.539 & 0.461 & 0.687 & 0.313 & 0.533 & 0.467 \\
$\mathrm{Cu}_{5} \mathrm{O}_{3} / \mathrm{UNCD}$ & 0.400 & 0.600 & 1.0 & 0.0 & 0.748 & 0.252 \\
$\mathrm{Cu}_{4} \mathrm{O}_{2} / \mathrm{Al}_{2} \mathrm{O}_{3}$ & 0.312 & 0.688 & 0.883 & 0.117 & 0.466 & 0.534 \\
$\mathrm{Cu}_{4} \mathrm{O}_{3} / \mathrm{Al}_{2} \mathrm{O}_{3}$ & 0.193 & 0.807 & 0.404 & 0.596 & 0.203 & 0.797 \\
$\mathrm{Cu}_{4} \mathrm{O}_{4} / \mathrm{Al}_{2} \mathrm{O}_{3}$ & 0.162 & 0.838 & 0.236 & 0.764 & 0.375 & 0.625 \\
$\mathrm{Cu}_{4} \mathrm{O}_{5} / \mathrm{Al}_{2} \mathrm{O}_{3}$ & 0.111 & 0.889 & 0.245 & 0.755 & 0.246 & 0.754 \\
\hline
\end{tabular}

Table S3. Obtained coefficients of $\mathrm{Cu}_{4} \mathrm{O}_{\mathrm{x}} / \mathrm{Al}_{2} \mathrm{O}_{3}(\mathrm{x}=2-5)$ from LCF to the experimental XANES at $423 \mathrm{~K}$, $673 \mathrm{~K}$, and $773 \mathrm{~K} . \mathrm{Cu}_{4} \mathrm{O}_{2}$ becomes dominant at high temperature showing a reduction in the cluster oxidation state.

\begin{tabular}{ccccc}
\hline $\mathbf{T}(\mathbf{K})$ & $\mathbf{C}_{\mathbf{C u} 402}$ & $\mathbf{C}_{\mathbf{C u} 403}$ & $\mathbf{C}_{\mathbf{C u} 404}$ & $\mathbf{C}_{\mathbf{C u} 405}$ \\
\hline 423 & 0.154 & 0.0 & 0.499 & 0.347 \\
673 & 0.390 & 0.0 & 0.510 & 0.100 \\
773 & 0.706 & 0.0 & 0.261 & 0.03 \\
\hline
\end{tabular}

Table S4. Energies of $\mathrm{Cu}_{4} \mathrm{O}_{\mathrm{x}} / \mathrm{Al}_{2} \mathrm{O}_{3}(\mathrm{x}=2-5), \mathrm{Cu}_{5} \mathrm{O}_{5} / \mathrm{UNCD}$, and $\mathrm{Cu}_{5} \mathrm{O}_{3} / \mathrm{UNCD}$ obtained from PES sampling. The free energies of $\mathrm{Cu}_{4} \mathrm{O}_{\mathrm{x}} / \mathrm{Al}_{2} \mathrm{O}_{3}(\mathrm{x}=2-5)$ are calculated at $p_{\mathrm{O} 2}=0.5$ bar and $\mathrm{T}=473 \mathrm{~K}$ and with respect to the global minimum structure $\left(\mathrm{Cu}_{4} \mathrm{O}_{3} / \mathrm{Al}_{2} \mathrm{O}_{3}-\mathrm{I}\right)$.

\begin{tabular}{ccc}
\hline Structure & $\mathbf{E}(\mathbf{e V})$ & $\mathbf{G}(\mathbf{e V})$ \\
\hline $\mathrm{Cu}_{4} \mathrm{O}_{2} / \mathrm{Al}_{2} \mathrm{O}_{3}-\mathrm{I}$ & 0 & 1.40 \\
$\mathrm{Cu}_{4} \mathrm{O}_{2} / \mathrm{Al}_{2} \mathrm{O}_{3}$-II & 1.34 & 2.74 \\
$\mathrm{Cu}_{4} \mathrm{O}_{2} / \mathrm{Al}_{2} \mathrm{O}_{3}$-III & 2.42 & 3.82 \\
\hline
\end{tabular}




\begin{tabular}{ccc}
\hline $\mathrm{Cu}_{4} \mathrm{O}_{3} / \mathrm{Al}_{2} \mathrm{O}_{3}-\mathrm{I}$ & 0 & 0 \\
$\mathrm{Cu}_{4} \mathrm{O}_{3} / \mathrm{Al}_{2} \mathrm{O}_{3}$-II & 0.15 & 0.15 \\
$\mathrm{Cu}_{4} \mathrm{O}_{3} / \mathrm{Al}_{2} \mathrm{O}_{3}$-III & 0.39 & 0.39 \\
\hline $\mathrm{Cu}_{4} \mathrm{O}_{4} / \mathrm{Al}_{2} \mathrm{O}_{3}$-I & 0 & 0.58 \\
$\mathrm{Cu}_{4} \mathrm{O}_{4} / \mathrm{Al}_{2} \mathrm{O}_{3}$-II & 0.22 & 0.80 \\
$\mathrm{Cu}_{4} \mathrm{O}_{4} / \mathrm{Al}_{2} \mathrm{O}_{3}$-III & 0.44 & 1.02 \\
\hline $\mathrm{Cu}_{4} \mathrm{O}_{5} / \mathrm{Al}_{2} \mathrm{O}_{3}$-I & 0 & 1.07 \\
$\mathrm{Cu}_{4} \mathrm{O}_{5} / \mathrm{Al}_{2} \mathrm{O}_{3}$-II & 0.66 & 1.73 \\
$\mathrm{Cu}_{4} \mathrm{O}_{5} / \mathrm{Al}_{2} \mathrm{O}_{3}$-III & 1.92 & 2.99 \\
\hline $\mathrm{Cu}_{5} \mathrm{O}_{3} / \mathrm{UNCD} \mathrm{I}$ & 0 & - \\
$\mathrm{Cu}_{5} \mathrm{O}_{3} / \mathrm{UNCD}-\mathrm{II}$ & 0.49 & - \\
$\mathrm{Cu}_{5} \mathrm{O}_{3} / \mathrm{UNCD} \mathrm{III}$ & 1.39 & - \\
\hline $\mathrm{Cu}_{5} \mathrm{O}_{5} / \mathrm{UNCD}-\mathrm{I}$ & 0 & - \\
$\mathrm{Cu}_{5} \mathrm{O}_{5} / \mathrm{UNCD} \mathrm{II}$ & 0.06 & - \\
$\mathrm{Cu}_{5} \mathrm{O}_{5} / \mathrm{UNCD}-\mathrm{III}$ & 0.68 & - \\
\hline
\end{tabular}

